White, 21 (37.5\%) Asian, and 10 (18\%) Black. The risk was highest among Asian infants $(\mathrm{p}<0.001)$. An electroclinical syndrome was identified in $24(42 \%)$ cases of which 21 were epileptic encephalopathies (West [16], Ohtahara [2], and Dravet [3] syndromes). Overall, an underlying etiology for the epilepsy was identified in 29 children $(51 \%$ of the cohort). Developmental brain abnormalities (polymicrogyria, tuberous sclerosis) were most frequent, occurring in $11(21 \%)$, followed by acquired brain insults in $9(16 \%)$ infants. Acquired causes included meningitis in $5(9 \%)$, HIE in $4(7 \%)$, and metabolic disorder in 4 (7\%). Chromosomal abnormalities occurred in $4(7 \%)$. MR images of 51 cases showed abnormalities in $37(72 \%)$ and etiologically relevant abnormality in 26 (51\%). (Eltze CM, Chong WK, Cox T, et al. A population-based study of newly diagnosed epilepsy in infants. Epilepsia 2013 Mar;54(3):437-445). (Response: Dr Christin M Eltze, Neurosciences Unit, UCL-Institute of Child Health, 4/5 Long Yard, London WC1N 3LU, UK. E-mail: c.eltze@ucl.ac.uk).

COMMENT. Infantile onset epilepsy frequently presents with intractable seizures and is commonly associated with diffuse encephalopathy, metabolic or structural brain abnormalities. Identification of specific electroclinical syndromes at seizure onset requires specialist intervention with video-EEG recordings, MRI, metabolic, and genetic studies. The EEG associated with an epileptic encephalopathy is diffusely abnormal and varies with cerebral maturation. (Nordli DR Jr. Epileptic encephalopathies in infants and children. J Clin Neurophysiol 2012 Oct;29(5):420-4). Details of EEG findings are important in the workup and in the classification of infantile seizures. Early referral of infantile seizure patients to a Pediatric Epilepsy Center is usually indicated for accurate seizure classification and optimal management. (Alam S, Lux AL. Epilepsies in infancy. Arch Dis Child 2012 Nov;97(11):985-92).

\title{
GENETICS OF BENIGN FAMILIAL INFANTILE EPILEPSIES
}

Investigators at Instituto G Gaslini, Genova, Italy and multiple other centers in Italy studied the genetics of benign familial epilepsies of the first year of life and assessed the extent of the genetic overlap between neonatal and infantile seizure syndromes. Families with at least two first-degree relatives affected by focal seizures with onset in the first year of life and normal development before seizure onset were included. A total of 46 families including 165 affected members were collected and were classified as benign familial neonatal seizures (BFNS) in 8 families, benign familial neonatal-infantile seizures (BFNIS) in 9 (1-4 months of age at onset), and benign familial infantile seizures (BFIS) in 29 (onset after 4 months of age in all family members). Genetic analysis identified 41 mutations, 14 affecting KCNQ2, 1 affecting KCNQ3, 5 affecting SCN2A, and 21 affecting PRRT2. The detection rate of mutations in this cohort was $89 \%$. Mutations specifically involve KCNQ2 in BFNS, KCNQ2 (6 families) and SCN2A (two families) in BFNIS. BFIS families are most genetically heterogeneous, with all 4 genes involved, $70 \%$ carrying a PRRT2 mutation. PRRT2 mutations are clustered in families with BFIS and also, with paroxysmal kinesigenic dyskinesia.

KCNQ2 mutation is frequently represented in the entire spectrum of disorders, progressively decreasing with age, and may be predictive of afebrile seizures during follow-up, beyond the typical neonatal seizures. Age of onset of seizures is significantly 
correlated with genetics: $90 \%$ of BFNS families are linked to KCNQ2 compared to only $3 \%$ of BFIS families. (Zara F, Specchio N, Striano P, et al. Genetic testing in benign familial epilepsies of the first year of life: Clinical and diagnostic significance. Epilepsia 2013 Mar;54(3):425-36). (Response: Dr Federico Zara. E-mail: federicozara@ospedalegaslini.ge.it).

COMMENT. Mutational screening for neonatal and infantile seizures should involve KCNQ2 in both BFNS and BFNIS, and PRRT2 in BFIS families. A clear clinical classification of the seizure phenotype is an essential preliminary to genetic analysis. In addition to confirming a clinical diagnosis, a positive SCN1A mutation will influence treatment and improve seizure control. (Brunklaus A, et al. The clinical utility of an SCN1A genetic diagnosis in infantile-onset epilepsy. Dev Med Child Neurol 2013 Feb;55(2):154-61).

\section{ANTIEPILEPTIC DRUGS AND DIET}

\section{FETAL AED EXPOSURE AND COGNITIVE OUTCOME AT AGE 6}

Investigators at Emory University, Atlanta, GA and multiple centers in the USA and UK conducted a prospective study of the effects of antiepileptic drug (AED) monotherapy (carbamazepine, lamotrigine, phenytoin, or valproate) on the intelligence quotient (IQ) at 6 years of age (age-6 IQ). Of 305 mothers and 311 children (6 twin pairs) in the primary analysis, 224 children completed the 6 years of follow-up. Age-6 IQ was 7-10 points lower after exposure to valproate than to carbamazepine, lamotrigine, or phenytoin $(\mathrm{p}=0.0015,0.0003,0.0006$, respectively). Measures of verbal and memory abilities were lower in children exposed to valproate compared to the other AEDs, and non-verbal and executive functions were lower with valproate compared to lamotrigine (but not carbamazepine or phenytoin). High doses of valproate were negatively associated with IQ, verbal ability, non-verbal ability, memory, and executive function; other AEDs were not. Age-6 IQ correlated with IQs at younger ages, and IQ improved with age for infants exposed to any AED. Right-handedness was less frequent overall and in the lamotrigine and valproate groups. Verbal abilities were lower than non-verbal abilities overall and in the lamotrigine and valproate groups. Mean IQs were higher in children exposed to periconceptual folate than in unexposed children $(\mathrm{p}=0.0009)$. (Meador KJ, Baker GA, Browning N, et al, for the NEAD Study Group. Fetal antiepileptic drug exposure and cognitive outcomes at age 6 years (NEAD study): a prospective observational study. Lancet Neurol 2013 Mar;12(3):244-52). (Response: Prof Kimford J Meador, Dept of Neurology, Emory University, Atlanta, GA. E-mail: kimford.meador@emory.edu).

COMMENT. Fetal valproate exposure is associated with a range of cognitive deficits at 6 years of age, an effect dependent on the dose. IQ improves with age and with periconceptual folate. The authors hypothesize that a change in cerebral lateralization, with reduced right-handedness and lower verbal (vs non-verbal) abilities, may be caused by exposure to AEDs. 